\title{
ANÁLISE DE USABILIDADE DO SISTEMA Q-ACADÊMICO UTILIZANDO O MÉTODO SYSTEM USABILITY SCALE (SUS): UM ESTUDO DE CASO
}

\author{
Rodolfo Pereira da Costa ${ }^{1}$, Antonio Fernando Siqueira dos Santos ${ }^{1}$, Cynthia \\ Pinheiro Santiago ${ }^{1}$ \\ ${ }^{1}$ Ciência da Computação - IFCE - Instituto Federal de Educação, Ciência e Tecnologia \\ do Ceará - Campus Tianguá \\ Endereço
}

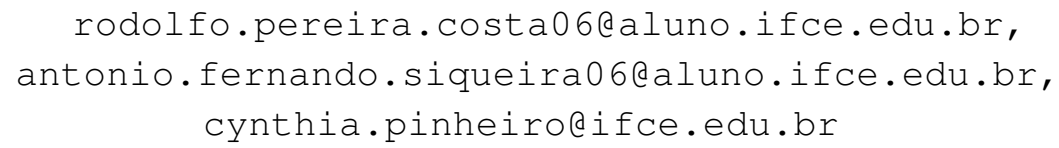

\begin{abstract}
Computerization in the academic community consists in the use of systems that help in the routines of students, teachers and administrative technicians, being able to facilitate processes, strengthening relationships between the parties and promoting the achievement of goals. For this to be effective, it is necessary that these systems have good usability and that they meet the expectations of their users, and it is necessary to provide an adequate interface. Thus, the work in question consists of analyzing the Q-academic system, a WEB system for academic management, through a usage analysis using the System Usability Scale (SUS) method in order to understand the perceptions of users data on the use of the system in question.
\end{abstract}

Keywords: Usability. Q-academic. SUS.

Resumo. A informatização na comunidade acadêmica consiste na utilização de sistemas que auxiliam nas rotinas dos estudantes, professores e técnicos administrativos, sendo capaz de facilitar os processos, estreitando as relações entre as partes e promovendo o alcance dos objetivos. Para que isso seja eficaz, é necessário que esses sistemas possuam boa usabilidade e que atendam as expectativas dos seus usuários, sendo necessário fornecer uma interface adequada. Dessa forma, o trabalho em questão consiste em analisar o sistema Q-acadêmico, um sistema WEB para gestão acadêmica, por meio de uma análise de usabilidade utilizando o método System Usability Scale (SUS) com o intuito de compreender as percepções dos usuários diante da utilização do sistema em questão.

Palavras-chave: Usabilidade. Q-acadêmico. SUS.

\section{Introdução}

Desde o surgimento da internet, são inúmeros os produtos e serviços que vem sendo desenvolvidos constantemente, entre eles estão os sistemas digitais que passaram a fazer parte da vida das pessoas, do trabalho ao lazer. É nesse cenário, que se torna fundamental que um produto digital seja interativo, utilizando métodos de usabilidade que posicione 
os usuários como foco principal. Dessa forma, observa-se a importância do conceito de usabilidade, pois está relacionado com a eficiência e eficácia das interfaces dos sistemas de informação. Este conceito quando é implementado corretamente passa a atuar como aspecto fundamental na promoção da qualidade e satisfação do usuário.

Steve Krug [KRUG, 2008] define Usabilidade como "assegurar-se de que algo funcione e bem: que uma pessoa com habilidade e experiência comuns possa usar algo para seu propósito desejado sem ficar frustrada com isso". Por outro lado, Nielsen [NIELSEN, 1993], trata Usabilidade como "um atributo de qualidade que relaciona o quanto algo é fácil de utilizar".

Em um ambiente acadêmico, os sistemas WEB auxiliam tanto na gestão administrativa como nos processos acadêmicos. É por essa razão que sua interface precisa ser capaz de garantir uma experiência adequada para seus usuários, pois ele funciona como uma ponte de ligação entre aluno, professor e pessoal administrativo, onde todos necessitam de suas funcionalidades cotidianamente.

É visando promover uma relação de proximidade entre corpo docente e discente que os Institutos Federais, fazem uso do sistema WEB Q-acadêmico, que por sua vez, foi projetado para facilitar a geração de informações e o controle destas dentro do campo institucional.

Entretanto, a preocupação do desenvolvimento destes sistemas não se resume apenas ao cumprimento das funções para quais ele foi projetado, mas também em como será a sua interatividade e garantia de experiência positiva aos seus usuários. Pois, quando se considera a usabilidade no processo do estudo para o desenvolvimento de interfaces WEB, várias falhas podem ser eliminadas.

O presente trabalho tem como objetivo analisar o Sistema Q-acadêmico por meio do método System Usability Scale - SUS [BROOK, 1996], que é realizado através de um questionário com perguntas direcionadas a análise de usabilidade, afim de levantar as percepções dos usuários diante da sua utilização.

\section{Referencial Teórico}

\subsection{Sistema WEB Q-acadêmico}

Um Sistema WEB é um software hospedado em um servidor na Internet, que tem como principal característica a possibilidade de poder ser acessado de qualquer lugar e de qualquer dispositivo, desde que este possua um navegador com acesso à internet. É por esse motivo que os Institutos Federais, pensando em facilitar o cotidiano dos alunos, disponibiliza o sistema WEB de gestão acadêmica Q-acadêmico.

Segundo o manual, o sistema Q-acadêmico "é uma moderna solução que se adéqua a realidade diária de alunos e professores. Através da internet, o professor e o aluno participam de atividades escolares de forma rápida e fácil" [QUALIDATA, 2005].

Com a principal finalidade de gerenciar as rotinas administrativas no campo acadêmico, o sistema Q-acadêmico foi projetado tanto para os professores, quando nos referimos a facilidade do controle e geração de informações, quanto para os alunos, no que se refere o acesso de funcionalidades que são necessárias para sua vivência na formação acadêmica. Dessa forma, o sistema em questão possui diversos módulos, sendo destinado aos 
professores e gestão administrativa, e alunos.

\subsection{Interação Humano-Computador - IHC}

Barbosa [BARBOSA et al., 2021]), define a Interação Humano-Computador como tudo o que acontece quando uma pessoa e um sistema computacional se unem para realizar tarefas, visando realizar um objetivo, ou seja, esta interação pode ser considerada como sendo um processo de manipulação, comunicação, conversa, troca, influência, e assim por diante.

Segundo [OLIVEIRA, 2011] a Interação Humano-Computador é uma área da computação que investiga o design, avalia e desenvolve interfaces para que usuários finais possam interagir com sistemas computacionais de uma forma eficiente e intuitiva.

O contexto em que o usuário está inserido e a forma como ele interpreta as informações apresentadas pela interface, é o que influencia na classificação da experiência obtida com o sistema, se foi boa ou ruim, por exemplo.

Portanto, não basta apenas o sistema garantir bom processamento e muito menos se limitar somente em cumprir sua função adequadamente, mas também deve promover a garantia da boa experiência do usuário, e isso, só é possível, garantindo a aplicação de diversos aspectos que estão voltados para as vertentes de usabilidade.

\subsection{Usabilidade}

Uma das primeiras definições foi feita por Shackel [SHACKEL, 1984], que descreveu usabilidade como uma propriedade não constante de um sistema ou equipamento e sua relação em função dos usuários, do treinamento e suporte que lhes foram dados, das tarefas executadas e dos ambientes onde as tarefas foram concluídas. Dito de outro modo, a usabilidade varia conforme o contexto considerado. Assim, um sistema ou um equipamento será considerado usável se for capaz de atender a uma determinada combinação de usuários, tarefas e ambientes.

Outra definição de usabilidade é sugerida pela Norma ISO 9241-11, que explica usabilidade como um conjunto de atributos de software relacionado ao esforço necessário para seu uso e para o julgamento individual de tal uso, por determinado conjunto de usuários [ISO, 1998].

A maneira como os humanos estão se relacionando com os ambientes digitais tem exigido cada vez mais a dinâmica da aplicação dos aspectos relativos à usabilidade, pois quando nos definimos este conceito, nos referimos aquilo que é fácil de usar, e é por esse motivo que devemos considerar as necessidades do utilizador e o contexto em que este está inserido. Dessa forma, Krug [KRUG, 2008] define o conceito usabilidade como a facilidade que um usuário, tem ao interagir com determinado sistema sem necessitar de muito esforço.

\subsubsection{SUS}

Uma abordagem popular e econômica para medição de usabilidade é o uso de pesquisas padronizadas. O SUS é uma das ferramentas mais utilizadas para medir a percepção de usabilidade de um sistema ou produto [FINSTAD, 2010]. O método SUS é bastante 
popular para laboratórios e testes de usabilidade não moderados, pois fornecem ideias sobre o que os usuários pensam sobre a usabilidade do produto ou site testado [SAURO e LEWIS, 2012].

O método de avaliação SUS conta com dez questões, onde os participantes podem responder em uma escala de um a cinco, onde estão descritas no questionário apresentado na Tabela 1 .

\section{Tabela 1. Questionário SUS traduzido para o português}

\begin{tabular}{|l|l|}
\hline Item & Item correspondente em português \\
\hline 1 & Acho que gostaria de utilizar este produto com frequência \\
\hline 2 & Eu acho o sistema desnecessariamente complexo \\
\hline 3 & Eu achei o sistema fácil de usar \\
\hline 4 & $\begin{array}{l}\text { Eu acho que precisaria de ajuda de uma pessoa com conhecimentos técnicos para usar } \\
\text { o sistema }\end{array}$ \\
\hline 5 & Eu acho que as várias funções do sistema estãomuito bem integradas \\
\hline 6 & Eu acho que o sistema apresenta muita inconsistência \\
\hline 7 & Eu imagino que as pessoas aprenderão como usar esse sistema rapidamente \\
\hline 8 & Eu achei o sistema atrapalhado de usar \\
\hline 9 & Eu me senti confiante ao usar o sistema \\
\hline 10 & Eu precisei aprender várias coisas novas antes de conseguir usar o sistema \\
\hline
\end{tabular}

A primeira etapa na pontuação de um SUS é determinar a contribuição da pontuação de cada item, ou seja, para questões identificadas por números ímpares, é subtraído 1 da pontuação que o usuário respondeu, para itens de números pares, é subtraído 5 da contribuição da pontuação do usuário. Ou seja, se a pontuação atribuída corresponder a 2, o resultado será 3. Para obter a pontuação geral do SUS, multiplique a soma dos resultados da pontuação dos itens por 2,5. Assim, as pontuações gerais do SUS variam de 0 a 100 .

Brooke [BROOK, 1996] ainda relata que o método SUS produz um único número que representa uma medida composta da usabilidade geral do sistema que está sendo estudado e que as pontuações de itens individuais não são significativas por si mesmas, necessitando assim, de dados de mais de 20 participantes, para sua respectiva validação.

\section{Trabalhos Relacionados}

Carvalho [CARVALHO, 2016], faz uso de uma combinação de técnicas de avaliação, com diferentes métodos para avaliar o mesmo sistema. O autor, então, realizou uma coleta de opiniões dos usuários do Sistema Integrado de Gestão de Atividades Acadêmicas (SIGAA) com o objetivo de perguntar aos alunos quais as principais funcionalidades que eles consideram mais difíceis de usar. Outra abordagem no trabalho em questão foi a utilização da Avaliação Heurística por possuir um ótimo custo-benefício e ser um dos mais utilizados. Para a realização da Avaliação Heurística foi selecionado o escopo de avaliação, ou seja, quais telas deveriam ser avaliadas no módulo de alunos do SIGAA. O 
autor salienta que para a realização da avaliação, foi escolhida as heurísticas de Nielsen, porque elas englobam todos os aspectos das boas práticas de usabilidade.

Grossi, Pisa \& Marin [GROSSI et al., 2014] realizaram um pesquisa aplicada de produção tecnológica destinada a desenvolver um aplicativo em ambiente web e tecnologia móvel para consulta de medicamentos quimioterápicos na auditoria de contas hospitalares. Para avaliar se o aplicativo criado se adequava as necessidades dos usuários, utilizou-se o questionário System Usability Scale (SUS) com enfermeiros auditores, e se estava adequado aos critérios de usabilidade. Em relação a satisfação, os enfermeiros consideraram o aplicativo fácil de ser utilizado, inovador, completo e com informações importantes. Além disso, um dos enfermeiros elogiou as abas de "estabilidade", "grupo farmacológico"e "compatibilidade com frasco e equipos"que são informações muito utilizadas na prática deles. Deste modo, a avaliação feita com o questionário SUS mostrou-se satisfatória, o que corrobora com outros estudos que também aplicaram esta ferramenta.

Já no estudo de [SALLES et al., 2015] foi realizada uma avaliação do sistema Q-acadêmico IFFluminense, por meio de uma amostra de alunos de todos os períodos e professores dos cursos de graduação (Tecnólogo e Bacharelado) da área de Informática. $\mathrm{O}$ instrumento utilizado para coleta das informações foi o questionário, onde foram avaliadas as seguintes características de qualidade do produto de software: Usabilidade, Funcionalidade, Eficiência e Confiabilidade. Foi elaborado um questionário dividido em três partes classificados como: Facilidade de Uso, Comportamento em Relação a Falhas, Eficiência e Satisfação das Necessidades. O trabalho buscou avaliar o sistema acadêmico em relação às opiniões de um grupo específico de usuários. O resultado das avaliações apresentou divergências de acordo com o perfil do usuário. O autor sugere que o sistema Q-acadêmico seja avaliado através de um modelo mais detalhado, onde os usuários possuam mais opções de critérios e alternativas para a avaliação. Este trabalho foi de suma importância, porque a avaliação de qualidade realizada pelos autores nos permitiu entender um pouco de quais as falhas e queixas dos usuários que o sistema Q-acadêmico desde então vinha enfrentando.

\section{Materiais e Métodos}

Com o propósito de realizar um estudo de caso sobre a usabilidade do sistema Q-acadêmico, a metodologia utilizada na presente pesquisa, possui uma abordagem quantitativa, utilizando um método empírico de avaliação em IHC, com intuito de analisar a usabilidade e investigar as percepções dos usuários acerca do sistema Q-acadêmico.

Para isso, foi aplicado um questionário por meio do Google Forms, o questionário foi dividido em duas partes: na primeira etapa, foram aplicadas perguntas para identificação dos perfis dos participantes, e posteriormente esses mesmos participantes participaram de uma avaliação que foi realizada utilizando o método SUS [BROOK, 1996], que tem por finalidade identificar os fatores que influenciam a satisfação do usuário frente à usabilidade.

Dessa forma, o principal intuito da aplicação do questionário é verificar através dos resultados obtidos por meio das respostas dos participantes da pesquisa, as percepções diante da utilização do Q-acadêmico. As seções do questionário foram divididas em três etapas, a primeira contava com a apresentação da pesquisa, seguido do termo de consentimento. Na segunda parte, foi possível coletar as informações do perfil do usuário. Posteriormente, os participantes, tiveram acesso ao questionário SUS [BROOK, 1996], no 
qual puderam responder os dez itens escolhendo de 1 a 5 , onde ao responder 1 , classificaria como discordo fortemente, e ao escolher 5, concordaria fortemente com a afirmação.

\section{Resultados}

Ao analisar um site por meio do método SUS é possível classificar a sua usabilidade entre 0 a 100, e quanto mais próximo de 100, maior é a satisfação do usuário.

Bangor [BANGOR et al., 2008], classifica a facilidade de uso de um site ou produto utilizando uma sequência gerada da esquerda para a direita como, Muito ruim; Ruim; OK; Bom; Excelente; Melhor imaginável. Assim, definindo uma escala de classificação em que pontua o SUS abaixo de 50 como um "F", entre 60 e 69 um "D", entre 70 e 79 um "C", entre 80 e 89 um "B" e 90 e acima um "A". Conforme Tabela 1 abaixo:

\begin{tabular}{|c|c|c|}
\hline SUS Score & Grade & Adjetivo \\
\hline $90.0-100$ & A & Melhor imaginável \\
\hline $80.0-89.9$ & B & Excelente \\
\hline $70.0-79.9$ & C & Bom \\
\hline $60.0-69.9$ & D & OK \\
\hline $50.0-59.9$ & E & Ruim \\
\hline$<49.9$ & F & Muito Ruim \\
\hline
\end{tabular}

Tabela 1. Classificação SUS de usabilidade segundo Bangor.

No entanto, Sauro [SAURO e LEWIS, 2012], organizou os dados do SUS por tipo de interface para gerar um benchmark global para o SUS, apresentada na Tabela 2, assim, combinando dados de Bangor [BANGOR et al., 2008], Sauro [SAURO e J, 2009] e Tullis e Albert [TULLIS e ALBERT, 2008]. Seu estudo, então, foi capaz de demonstrar o valor ponderado da média de todas as fontes, assim definindo como média o percentual de 68 com um desvio padrão de 12,5, ou seja, os sites que se encaixarem até esta média são considerados usáveis e classificados na escala com um "D”. Contudo, Sauro [SAURO e LEWIS, 2012] defende que em nota a curva de pontuação do SUS 68 está no centro da faixa de um "C". 


\begin{tabular}{|c|c|}
\hline SUS Score & Grade \\
\hline $84.1-100$ & A+ \\
\hline $80.0-84.0$ & A \\
\hline $78.9-80.7$ & A- \\
\hline $77.2-78.8$ & B+ \\
\hline $74.1-77.1$ & B \\
\hline $72.6-74.0$ & B- \\
\hline $71.1-72.5$ & C+ \\
\hline $65.0-71.0$ & C \\
\hline $62.7-64.9$ & C- \\
\hline $51.7-62.6$ & D \\
\hline$<51.7$ & F \\
\hline
\end{tabular}

Tabela 2. Classificação SUS de usabilidade segundo Sauro.

No caso deste trabalho, como mencionado anteriormente, foi possível coletar opiniões de 101 pessoas, onde puderam responder os 10 itens presentes na pesquisa, decidindo entre uma escala de 1 a 5, onde ao escolher 1 como resposta, discordaria totalmente, e 5, concordaria totalmente com a respectiva afirmação. Dessa forma, a Figura 3 mostra como os 101 participantes avaliaram o sistema Q-acadêmico de acordo com o método SUS.

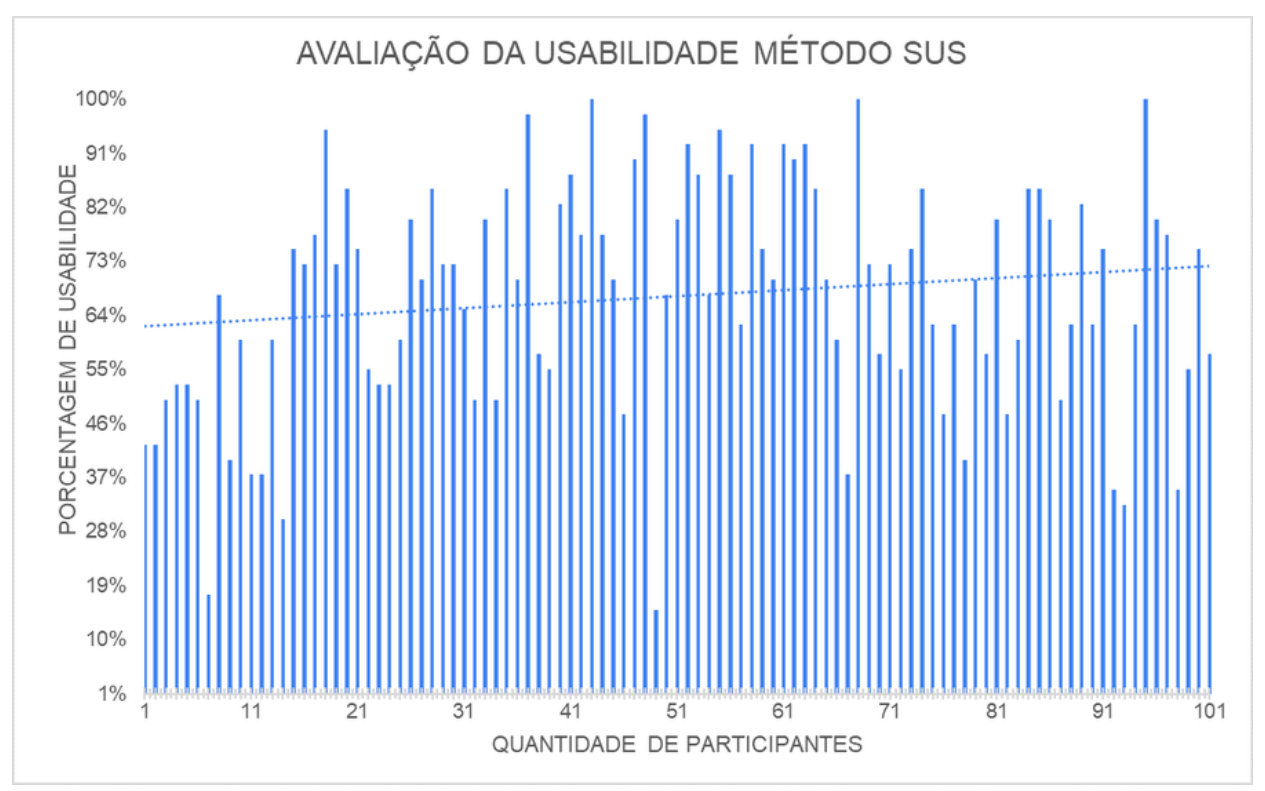

Figura 1. Avaliação de Usabilidade Método SUS

Como visto na Figura 1, foi realizado o cálculo SUS a partir da média de respostas dos 101 avaliadores, e este classificou o sistema Q-acadêmico com uma usabilidade de $67,22 \%$. Portanto, comparado ao benchmark global desenvolvido por Sauro [SAURO e LEWIS, 2012] o sistema em questão se encaixaria na escala "C" (Tabela 2) e para Bangor [BANGOR et al., 2008] um "D", sendo considerado um sistema com tendência para um "OK", demonstrado na Tabela 1. 


\section{Conclusão}

Este estudo teve como principal motivação, a análise da usabilidade do sistema Qacadêmico que é utilizado pela comunidade acadêmica do Instituto Federal de Educação, Ciência e Tecnologia do Ceará - IFCE Campus Tianguá, por meio da aplicação do questionário SUS [BROOK, 1996] que levanta informações relevantes relacionadas à identifcação do nível de usabilidade do sistema estudado. A avaliação contou com a participação de 101 usuários, que a partir das suas respostas classificaram o sistema em questão com uma usabilidade de 67,22\% (Figura 1). Nesse caso, segundo o benchmark global defendido por Bangor [BANGOR et al., 2008] e Sauro [SAURO e LEWIS, 2012] a usabilidade SUS [BROOK, 1996] do sistema Q-acadêmico se classifica como um sistema "OK".

Com esta avaliação foi possível coletar dados capazes de expressar o nível de usabilidade, considerando as impressões que o usuário adquire a partir do uso do sistema, servindo de base para mais estudos e futuras sugestões de melhorias.

\section{Referências}

BANGOR, A., KORTUN, P. T., e MILLER, J. T. (2008). The System Usability Scale (SUS): an Empirical evaluation. International Journal of Human-Computer Interaction

BARBOSA, S., SILVA, B. S. D., SILVEIRA, M., GASPARINI, I., DARIN, T., e BARBOSA, G. (2021). Interação humano-computador e experiência do usuário.

BROOK, J. (1996). A “quick and dirty"usability scale. Usability Evaluation in Industry. pages $189-194$.

CARVALHO, N. (2016). Avaliação de usabilidade do módulo de alunos do sistema integrado de gestão acadêmicas - sigaa/ufc: Um estudo de caso. Quixadá.

FINSTAD, K. (2010). The usability metric for user experience. Intel Corporation.

GROSSI, L. M., PISA, I. T., e MARIN, H. D. F. (2014). Oncoaudit: desenvolvimento e avaliação de aplicativo para enfermeiros auditores.

ISO (1998). Ergonomic requirements for office work with visual display terminals (vdts). Part 11: Guidance on usability.

KRUG, S. (2008). Não me faça pensar: Uma abordagem de bom senso à usabilidade na web.

NIELSEN, J. (1993). Usability engineering. MA: Academic Press. Boston.

OLIVEIRA, I. (2011). A interação homem-computador através dos tempos.

QUALIDATA (2005). Manual 2.0. Módulo Web - Aluno.

SALLES, A. R. D. A., S NETO, C. M., DA, C. P., e SILVA, J. D. (2015). Avaliação da qualidade de um sistema acadêmico: Estudo de caso no q-acadêmico. CITI 2015.

SAURO, J. e J, L. (2009). Correlations among prototypical usability metrics: Evidence for the construct of usability. Proceedings of CHI 2009, pages $1609-1618$.

SAURO, J. e LEWIS, J. (2012). Quantifying the user experience practical statistics for user research.

SHACKEL, B. (1984). The concept of usability. Englewood Cliffs.

TULLIS, T. e ALBERT, W. (2008). Measuring the user experience. Amsterdam. 